\title{
On the Capacity of Memoryless Finite-State Multiple-Access Channels With Asymmetric State Information at the Encoders
}

\author{
Giacomo Como and Serdar Yüksel
}

\begin{abstract}
A single-letter characterization is provided for the capacity region of finite-state multiple-access channels, when the channel state process is an independent and identically distributed sequence, the transmitters have access to partial (quantized) state information, and complete channel state information is available at the receiver. The partial channel state information is assumed to be asymmetric at the encoders. As a main contribution, a tight converse coding theorem is presented. The difficulties associated with the case when the channel state has memory are discussed and connections to decentralized stochastic control theory are presented.
\end{abstract}

Index Terms-Asymmetric channel state information, decentralized stochastic control, multiple-access channel, nonnested information structure.

\section{INTRODUCTION AND LITERATURE REVIEW}

W IRELESS communication channels and Internet type networks are examples of channels where the channel characteristics are time-varying. In wireless channels, the mobility of users and changes in landscape as well as interference may lead to temporal variations in the channel quality. In network applications, user demand and node failure may affect the channel reliability. Such channel variation models may include fast fading and slow fading; in fast fading, the channel state is assumed to be changing for each use of the channel. On the other hand, in slow fading, the channel is assumed to be constant for each finite block length.

In such problems, the channel state can be transmitted to the encoders either explicitly, or through output feedback. Typically the feedback is not perfect, that is the encoder has only partial information regarding the state or the output variables. The present paper studies a particular case, finite-state multiple-access channels (MACs), where partial channel state information (CSI) is provided to the encoders causally. What makes such setup particularly interesting is the fact that the partial CSI available to the two transmitters is in general asymmetric, i.e., none

Manuscript received June 19, 2009; revised June 22, 2010; accepted September 24, 2010. Date of current version February 18, 2011. The work of S. Yüksel was supported by the Natural Sciences and Engineering Research Council of Canada (NSERC).

G. Como is with the Laboratory for Information and Decision Systems, Massachusetts Institute of Technology, Cambridge, MA 02139 USA (e-mail: giacomo@mit.edu).

S. Yüksel is with the Mathematics and Engineering Program, Department of Mathematics and Statistics, Queen's University, Kingston, Ontario, Canada, K7L 3N6 (e-mail: yuksel@mast.queensu.ca).

Communicated by E. Erkip, Associate Editor for Shannon Theory.

Digital Object Identifier 10.1109/TIT.2011.2104570 of the transmitters' CSI contains the CSI available to the other one. On the other hand, we assume that the receiver has access to perfect state information.

A single-letter characterization of the capacity region is provided for the case of independent and identically distributed (i.i.d.) channel state sequences. As we shall review shortly, results in the literature have already provided achievability results for such problems. The main contribution of this paper consists in providing a tight converse theorem. Our proof involves showing that restricting to encoders using only the quantized CSI on the current state does not cause any loss of optimality with respect to the most general class of admissible encoders using all the quantized CSI causally observed until a given time.

The problem at hand can be thought of as a decentralized stochastic control problem. We shall elaborate on this connection in the concluding section, where we shall also discuss in what our arguments fail when trying to extend them to a proof of the converse theorem for finite-state MACs with memory, and asymmetric CSI at the transmitters.

Let us now present a brief literature review. Capacity with partial channel state information at the transmitter is related to the problem of coding with unequal side information at the encoder and the decoder. The capacity of memoryless channels, with various cases of state information being available at neither, either or both the transmitter and receiver, has been studied in [13] and [7]. Reference [14] develops a stochastic control framework for the computation of the capacity of channels with memory and complete noiseless output feedback via the properties of the directed mutual information. Reference [8] considers fading channels with perfect channel state information at the transmitter, and shows that with instantaneous and perfect state information, the transmitter can adjust the data rates for each channel state to maximize the average transmission rate. Viswanathan [17] relaxes this assumption of perfect instantaneous state information, and studies the capacity of Markovian channels with delayed information. Reference [4] studies the capacity of Markov channels with perfect causal state information. The capacity of Markovian, finite-state channels with quantized state information available at the transmitter is studied in [20].

The works most closely related to ours are [6] and [9]. In [6], the capacity of general finite-state MACs with different levels of causal CSI at the transmitters is characterized in terms of multiletter formulas. Moreover, single-letter characterizations are provided for the capacity of finite-state MACs when the decoder has perfect CSI and the encoders are restricted to use only a finite window of, possibly limited, CSI; the capacity re- 


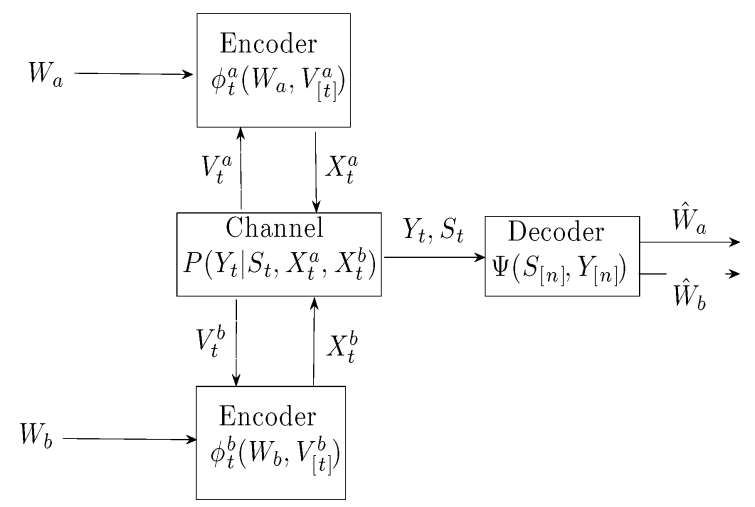

Fig. 1. Finite-state multiple-access channel with asymmetric partial state information at the transmitters.

gion without any such restriction is recovered in the limit of large window size. Reference [9] develops a general framework for approximating, and possibly characterizing, the capacity of channels with causal, and noncausal CSI: in particular, Theorem 4 therein provides a single-letter characterization of the capacity region of a MAC with independent CSI at the transmitters. With respect to [6] and [9], the present paper considers the somewhat simpler case of a MAC with i.i.d. state, where the encoders have causal, asymmetric, partial CSI, which is obtained through fixed quantizers acting componentwise. In contrast to [6], a single-letter expression for the capacity region is obtained in this case without any finite window restriction on the CSI available to the transmitters, while, differently from [9], the CSI available to the transmitters is not assumed to be independent. Recent related work also includes [11], providing an infinite-dimensional characterization for the capacity region for Multiple Access Channels with feedback, and [3], studying the case of MAC channels where the encoders have access to coded noncausal state information.

The rest of the paper is organized as follows. In Section II a formal statement of the problem and the main results are presented, consisting in a single-letter characterization of the capacity region of finite-state MACs with i.i.d. state. Section III contains the proof of achievability of the capacity region, while Section IV presents a proof of the converse coding theorem. Finally, in Section V, we discuss the issues arising when trying to generalize our arguments to the memory case, and present some final remarks on the connections of this problem with the decentralized stochastic control literature.

\section{CAPaCity OF I.I.D. Finite-STate MAC With Asymmetric PARTIAL CSI}

In the following, we shall present some notation, before formally stating the problem. For a vector $v$, and a positive integer $i, v_{i}$ will denote the $i$-th entry of $v$, while $v_{[i]}=\left(v_{1}, \ldots, v_{i}\right)$ will denote the vector of the first $i$ entries of $v$. Following a common convention, capital letters will be used to denote random variables (r.v.s), and small letters denote particular realizations. We shall use the standard notation $\mathrm{H}(\cdot)$, and $\mathrm{I}(\cdot ; \cdot)$ (respectively $\mathrm{H}(\cdot \mid \cdot)$, and $\mathrm{I}(\cdot ; \cdot \mid \cdot)$ ) for the (conditional) entropy and mutual information of r.v.s. With a slight abuse of notation, for
$0 \leq x \leq 1$, we shall write $\mathrm{H}(x)$ for the entropy of $x$. For a finite set $\mathcal{A}, \mathcal{P}(\mathcal{A})$ will denote the simplex of probability distributions over $\mathcal{A}$. Finally, for a positive integer $n$, we shall denote by $\mathcal{A}^{(n)}:=\bigcup_{0<s<n} \mathcal{A}^{s}$ the set of $\mathcal{A}$-strings of length smaller than $n$. $^{1}$

We shall consider a finite-state MAC with two transmitters, indexed by $i \in\{a, b\}$, and one receiver. Transmitter $i$ aims at reliably communicating a message $W_{i}$, uniformly distributed over some finite message set $\mathcal{W}_{i}$, to the receiver. The two messages $W_{a}$ and $W_{b}$ are assumed to be mutually independent. We shall use the notation $W:=\left(W_{a}, W_{b}\right)$ for the vector of the two messages.

The channel state process is modeled by a sequence $S=$ $\left\{S_{t}: t=1,2, \ldots\right\}$ of independent, identically distributed (i.i.d.) r.v.s, taking values in some finite-state space $\mathcal{S}$, and independent from $W$; the probability distribution of any $S_{t}$ is denoted by $P(\cdot) \in \mathcal{P}(\mathcal{S})$. The two encoders have access to causal, partial state information: at each time $t \geq 1$, encoder $i$ observes $V_{t}^{(i)}=q_{i}\left(S_{t}\right)$, where $q_{i}: \mathcal{S} \rightarrow \mathcal{V}_{i}$ is a quantizer modeling the imperfection in the state information. We shall denote by $V_{t}:=\left(V_{t}^{(a)}, V_{t}^{(b)}\right)$ the vector of quantized state observations, taking values in $\mathcal{V}:=\mathcal{V}_{a} \times \mathcal{V}_{b}$. The channel input of encoder $i$ at time $t, X_{t}^{(i)}$, takes values in a finite set $\mathcal{X}_{i}$, and is assumed to be a function of the locally available information $\left(W_{i}, V_{[t]}^{(i)}\right)$. The symbol $X_{t}=\left(X_{t}^{(a)}, X_{t}^{(b)}\right)$ will be used for the vector of the two channel inputs at time $t$, taking values in $\mathcal{X}:=\mathcal{X}_{a} \times \mathcal{X}_{b}$. The channel output at time $t, Y_{t}$, takes values in a finite set $\mathcal{Y}$; its conditional distribution satisfies

$$
\begin{aligned}
\mathbb{P}\left(Y_{t}=y \mid W=w, X_{[t]}=x_{[t]}, S_{[t]}=\right. & \left.s_{[t]}\right) \\
& =P\left(y_{t} \mid s_{t}, x_{t}\right)
\end{aligned}
$$

where, for any $s \in \mathcal{S}$, and $x \in \mathcal{X}, P(\cdot \mid s, x) \in \mathcal{P}(\mathcal{Y})$ is an output probability distribution. Finally, the decoder is assumed to have access to perfect causal state information (which may be known causally or noncausally); the estimated message pair will be denoted by $\hat{W}=\left(\hat{W}_{a}, \hat{W}_{b}\right)$.

We now present the class of transmission systems.

Definition 1: For a rate pair $R=\left(R_{a}, R_{b}\right)$, a block-length $n \geq 1$, and a target error probability $\varepsilon \geq 0$, an $(R, n, \epsilon)$-coding scheme consists of two sequences of functions

$$
\left\{\phi_{t}^{(i)}: \mathcal{W}_{i} \times \mathcal{V}_{i}^{t} \rightarrow \mathcal{X}_{i}\right\}_{1 \leq t \leq n},
$$

and a decoding function

$$
\psi: \mathcal{S}^{n} \times \mathcal{Y}^{n} \rightarrow \mathcal{W}_{a} \times \mathcal{W}_{b}
$$

such that, for $i \in\{a, b\}, 1 \leq t \leq n$ :

- $\left|\mathcal{W}_{i}\right| \geq 2^{R_{i} n}$

- $X_{t}^{(i)}=\phi_{t}^{(i)}\left(W_{i}, V_{[t]}^{(i)}\right)$

- $\hat{W}:=\psi\left(S_{[n]}, Y_{[n]}\right)$

- $\mathbb{P}(\hat{W} \neq W) \leq \varepsilon$.

We now proceed with the characterization of the capacity region.

${ }^{1}$ This includes the empty string, conventionally assumed to be the only element of $\mathcal{A}^{0}$. 
Definition 2: A rate pair $R=\left(R_{a}, R_{b}\right)$ is achievable if, for all $\varepsilon>0$, there exists, for some $n \geq 1$, an $(R, n, \varepsilon)$-coding scheme. The capacity region of the finite-state MAC is the closure of the set of all achievable rate pairs.

We now introduce what we call memoryless stationary team policies and their associated rate regions.

Definition 3: A memoryless stationary team policy is a family

$$
\pi=\left\{\pi_{i}\left(\cdot \mid v_{i}\right) \in \mathcal{P}\left(\mathcal{X}_{i}\right) \mid i \in\{a, b\}, v_{i} \in \mathcal{V}_{i}\right\}
$$

of probability distributions on the two channel input sets conditioned on the quantized observation of each transmitter. For every memoryless stationary team policy $\pi, \mathcal{R}(\pi)$ will denote the region of all rate pairs $R=\left(R_{a}, R_{b}\right)$ satisfying

$$
\begin{aligned}
& 0 \leq R_{a}<\mathrm{I}\left(X_{a} ; Y \mid X_{b}, S\right) \\
& 0 \leq R_{b}<\mathrm{I}\left(X_{b} ; Y \mid X_{a}, S\right) \\
& 0 \leq R_{a}+R_{b}<\mathrm{I}(X ; Y \mid S),
\end{aligned}
$$

where $S, X=\left(X_{a}, X_{b}\right)$, and $Y$, are r.v.s taking values in $\mathcal{S}$, $\mathcal{X}$, and $\mathcal{Y}$, respectively, and whose joint probability distribution

$$
\nu(s, x, y):=P(S=s, X=x, Y=y)
$$

factorizes as

$$
\nu(s, x, y)=P(s) \pi_{a}\left(x_{a} \mid q_{a}(s)\right) \pi_{b}\left(x_{b} \mid q_{b}(s)\right) P(y \mid s, x)
$$

We can now state the main result of the paper.

Theorem 4: The achievable rate region is given by

$$
\overline{\mathrm{co}}\left(\bigcup_{\pi} \mathcal{R}(\pi)\right),
$$

the closure of the convex hull of the rate regions associated to all possible memoryless stationary team policies $\pi$ as in (2). In Section III we shall prove the direct part of Theorem 4, namely that every rate pair $R \in \overline{\mathrm{co}}\left(\cup_{\pi} \mathcal{R}(\pi)\right)$ is achievable. In Section IV we shall prove the converse part, i.e., that no rate pair $R \in \mathbb{R}_{+}^{2} \backslash \overline{\mathrm{CO}}\left(\cup_{\pi} \mathcal{R}(\pi)\right)$ is achievable.

\section{ACHIEVABILITY OF THE CAPACITY REgION}

The result on achievability is known, and follows, e.g., from [6]. For convenience, we briefly sketch a different approach, as suggested at the beginning of [9Sect. VI]. The main idea consists in considering an equivalent MAC having as input mappings form the CSI information available at the transmitters to the original MAC's input. Specifically, one considers an equivalent memoryless MAC having output space $\mathcal{Z}:=\mathcal{S} \times \mathcal{Y}$ coinciding with the product of the state and output space of the original MAC, input spaces $\mathcal{U}_{i}:=\left\{u_{i}: \mathcal{V}_{i} \rightarrow \mathcal{X}_{i}\right\}$, for $i \in\{a, b\}$, and transition probabilities

$$
Q\left(z \mid u_{a}, u_{b}\right):=P(s) P\left(y \mid u_{a}\left(q_{a}(s)\right), u_{b}\left(q_{b}(s)\right)\right),
$$

where $z=(s, y)$. Then, a standard arguments shows that the rate region

$$
\begin{aligned}
R_{a} & <\mathrm{I}\left(U_{a} ; Z \mid U_{b}\right) \\
& R_{b}<\mathrm{I}\left(U_{b} ; Z \mid U_{a}\right) \\
R_{a}+R_{b} & <\mathrm{I}(U ; Z),
\end{aligned}
$$

is achievable on this MAC, where $U=\left(U_{a}, U_{b}\right)$ and $Z$ are random variables whose joint distribution factorizes as

$$
P\left(U_{a}, U_{b}, Z\right)=\mu_{a}\left(U_{a}\right) \mu_{b}\left(U_{b}\right) Q\left(Z \mid U_{a}, U_{b}\right),
$$

for some $\mu_{a} \in \mathcal{P}\left(\mathcal{U}_{a}\right)$, and $\mu_{b} \in \mathcal{P}\left(\mathcal{U}_{b}\right)$. Now, one can restrict himself to choosing probability distributions $\mu_{i}$ in $\mathcal{P}\left(\mathcal{U}_{i}\right)=$ $\mathcal{P}\left(\mathcal{X}_{i}^{\mathcal{S}_{i}}\right)$ with the product structure

$$
\mu_{i}\left(u_{i}\right)=\prod_{v_{i} \in \mathcal{V}_{i}} \pi_{i}\left(u_{i}\left(v_{i}\right) \mid v_{i}\right), \quad u_{i}: \mathcal{V}_{i} \rightarrow \mathcal{X}_{i},
$$

where $i \in\{a, b\}$, and $\pi$ is some memoryless stationary team policy, as in (2). Then, to any triple of r.v.s $\left(U_{a}, U_{b}, Z\right)$, with joint distribution as in (6), one can naturally associate random variables $S, X_{a}:=U_{a}\left(q_{a}(S)\right), X_{b}:=U_{b}\left(q_{b}(S)\right)$, and $Y$, whose joint probability distribution satisfies (4). Moreover, it can be readily verified that

$$
\begin{aligned}
\mathrm{I}\left(X_{a} ; Y \mid S, X_{b}\right) & =\mathrm{I}\left(U_{a} ; Z \mid U_{b}\right) \\
\mathrm{I}\left(X_{b} ; Y \mid S, X_{a}\right) & =\mathrm{I}\left(U_{b} ; Z \mid U_{a}\right) \\
\mathrm{I}(X ; Y \mid S) & =\mathrm{I}(U ; Z) .
\end{aligned}
$$

Hence, if a rate pair $R=\left(R_{a}, R_{b}\right)$ belongs to the rate region $\mathcal{R}(\pi)$ associated to some memoryless stationary team policy $\pi$ (i.e., if it satisfies (3)), then $R$ satisfies (5) for the product probability distributions $\mu_{a}, \mu_{b}$ defined by (7). This in turn implies that the rate pair is achievable on the original finite-state MAC $P$. The proof of achievability of the capacity region $\overline{\mathrm{CO}}\left(\cup_{\pi} \mathcal{R}(\pi)\right)$ then follows from a standard time-sharing principle (see, e.g., [5, Lemma 2.2, p.272]).

\section{Converse to the Coding Theorem}

In this section, we shall prove that no rate outside $\overline{\mathrm{CO}}\left(\cup_{\pi} \mathcal{R}(\pi)\right)$ is achievable. Lemma 5 shows that any achievable rate pair can be approximated by convex combinations of (conditional) mutual information terms. For $\varepsilon \in[0,1]$, define

$$
\eta(\varepsilon):=\frac{\varepsilon}{1-\varepsilon} \log |\mathcal{Y}|+\frac{\mathrm{H}(\varepsilon)}{1-\varepsilon},
$$

and observe that

$$
\lim _{\varepsilon \rightarrow 0} \eta(\varepsilon)=0 .
$$

For every $t \geq 1$, and $\boldsymbol{\sigma} \in \mathcal{S}^{t-1}$, define

$$
\alpha_{\boldsymbol{\sigma}}:=\frac{1}{n} \mathbb{P}\left(S_{[t-1]}=\boldsymbol{\sigma}\right) .
$$

Clearly, $\alpha_{\boldsymbol{\sigma}} \geq 0$, and

$$
\sum_{\boldsymbol{\sigma} \in \mathcal{S}^{(n)}} \alpha_{\boldsymbol{\sigma}}=\frac{1}{n} \sum_{1 \leq t \leq n} \sum_{\boldsymbol{\sigma} \in \mathcal{S}^{t-1}} \mathbb{P}\left(S_{[t-1]}=\boldsymbol{\sigma}\right)=1 .
$$


Lemma 5: For a rate pair $R \in \mathbb{R}_{+}^{2}$, a block-length $n \geq 1$, and a constant $\varepsilon \in(0,1 / 2)$, assume that there exists a $(R, n, \varepsilon)$-code. Then,

$$
\begin{aligned}
& R_{a}+R_{b} \leq \sum_{\boldsymbol{\sigma} \in \mathcal{S}^{(n)}} \alpha_{\boldsymbol{\sigma}} \mathrm{I}\left(X_{t} ; Y_{t} \mid S_{t}, S_{[t-1]}=\boldsymbol{\sigma}\right)+\eta(\varepsilon) \\
& R_{a} \leq \sum_{\boldsymbol{\sigma} \in \mathcal{S}^{(n)}} \alpha_{\boldsymbol{\sigma}} \mathrm{I}\left(X_{t}^{(a)} ; Y_{t} \mid X_{t}^{(b)}, S_{t}, S_{[t-1]}=\boldsymbol{\sigma}\right)+\eta(\varepsilon) . \\
& R_{b} \leq \sum_{\boldsymbol{\sigma} \in \mathcal{S}^{(n)}} \alpha_{\boldsymbol{\sigma}} \mathrm{I}\left(X_{t}^{(b)} ; Y_{t} \mid X_{t}^{(a)}, S_{t}, S_{[t-1]}=\boldsymbol{\sigma}\right)+\eta(\varepsilon) .
\end{aligned}
$$

Proof: By Fano's inequality we have the following estimate of the residual uncertainty on the messages given the full decoder's observation

$$
H\left(W \mid Y_{[n]} ; S_{[n]}\right) \leq \mathrm{H}(\varepsilon)+\varepsilon \log \left(\left|\mathcal{W}_{a}\right|\left|\mathcal{W}_{b}\right|\right) .
$$

For $1 \leq t \leq n$, we consider the conditional mutual information term

$$
\Delta_{t}:=\mathrm{I}\left(W ; Y_{t}, S_{t+1} \mid Y_{[t-1]}, S_{[t]}\right),
$$

and observe that

$$
\begin{aligned}
\sum_{1 \leq t \leq n} \Delta_{t} & =\mathrm{H}\left(W \mid S_{1}\right)-\mathrm{H}\left(W \mid S_{[n+1]}, Y_{[n]}\right) \\
& =\log \left(\left|\mathcal{W}_{a}\right|\left|\mathcal{W}_{b}\right|\right)-\mathrm{H}\left(W \mid S_{[n]}, Y_{[n]}\right),
\end{aligned}
$$

since the initial state $S_{1}$ is independent of the message pair $W$, and the final state $S_{n+1}$ is conditionally independent of $W$ given $\left(S_{[n]}, Y_{[n]}\right)$. On the other hand, using the conditional independence of $W$ from $S_{t+1}$ given $\left(S_{[t]}, Y_{[t]}\right)$, one gets

$$
\begin{aligned}
\Delta_{t} & =\mathrm{I}\left(W ; Y_{t}, S_{t+1} \mid Y_{[t-1]}, S_{[t]}\right) \\
& =\mathrm{I}\left(W ; Y_{t} \mid Y_{[t-1]}, S_{[t]}\right) \\
& =\mathrm{H}\left(Y_{t} \mid Y_{[t-1]}, S_{[t]}\right)-\mathrm{H}\left(Y_{t} \mid W, Y_{[t-1]}, S_{[t]}\right) \\
& \leq \mathrm{H}\left(Y_{t} \mid S_{[t]}\right)-\mathrm{H}\left(Y_{t} \mid W, S_{[t]}\right) \\
& =\mathrm{I}\left(W ; Y_{t} \mid S_{[t]}\right),
\end{aligned}
$$

where the above inequality follows from the fact that $\mathrm{H}\left(Y_{t}\right.$ | $\left.Y_{[t-1]}, S_{[t]}\right) \leq \mathrm{H}\left(Y_{t} \mid S_{[t]}\right)$, since removing the conditioning does not decrease the entropy, while $\mathrm{H}\left(Y_{t} \mid W, Y_{[t-1]}, S_{[t]}\right)=$ $\mathrm{H}\left(Y_{t} \mid W, S_{[t]}\right)$, as $Y_{t}$ is conditionally independent from $Y_{[t-1]}$ given $\left(W, S_{[t]}\right)$, due to the absence of output feedback. Since $\left(W, S_{[t]}\right)-\left(X_{t}, S_{t}\right)-Y_{t}$ forms a Markov chain, the data processing inequality implies that

$$
\mathrm{I}\left(W ; Y_{t} \mid S_{[t]}\right) \leq \mathrm{I}\left(X_{t} ; Y_{t} \mid S_{[t]}\right) .
$$

By combining (16), (17), (18), and (19), we then get

$$
\begin{aligned}
R_{a}+R_{b} & \leq \frac{1}{n} \log \left(\left|\mathcal{W}_{a}\right|\left|\mathcal{W}_{b}\right|\right) \\
& \leq \frac{1}{1-\varepsilon} \frac{1}{n} \sum_{1 \leq t \leq n} \mathrm{I}\left(X_{t} ; Y_{t} \mid S_{[t]}\right)+\frac{\mathrm{H}(\varepsilon)}{n(1-\varepsilon)} \\
& \leq \frac{1}{n} \sum_{1 \leq t \leq n} \mathrm{I}\left(X_{t} ; Y_{t} \mid S_{[t]}\right)+\eta(\varepsilon)
\end{aligned}
$$

Moreover, observe that

$$
\begin{aligned}
\mathrm{I}\left(X_{t} ; Y_{t} \mid S_{[t]}\right) & =\sum_{\boldsymbol{\sigma} \in \mathcal{S}^{t-1}} \mathbb{P}\left(S_{[t-1]}=\boldsymbol{\sigma}\right) \chi_{\boldsymbol{\sigma}} \\
& =n \sum_{\boldsymbol{\sigma} \in \mathcal{S}^{t-1}} \alpha_{\boldsymbol{\sigma}} \chi_{\boldsymbol{\sigma}}
\end{aligned}
$$

where $\chi_{\boldsymbol{\sigma}}:=\mathrm{I}\left(X_{t} ; Y_{t} \mid S_{t}, S_{[t-1]}=\boldsymbol{\sigma}\right)$. Substituting into (20) yields (13).

Analogously, let us focus on encoder $a$ : by Fano's inequality, we have that

$$
\mathrm{H}\left(W_{a} \mid Y_{[n]}, S_{[n]}\right) \leq \mathrm{H}(\varepsilon)+\varepsilon \log \left(\left|\mathcal{W}_{a}\right|\right) .
$$

For $t \geq 1$, define

$$
\Delta_{t}^{(a)}:=\mathrm{I}\left(W_{a} ; Y_{t}, S_{t+1} \mid W_{b}, Y_{[t-1]}, S_{[t]}\right),
$$

and observe that

$$
\begin{aligned}
\sum_{1 \leq t \leq n} \Delta_{t}^{(a)} & =\mathrm{H}\left(W_{a} \mid S_{1}, W_{b}\right)-\mathrm{H}\left(W_{a} \mid W_{b}, S_{[n+1]}, Y_{[n]}\right) \\
& \geq \log \left|\mathcal{W}_{a}\right|-\mathrm{H}\left(W_{a} \mid S_{[n]}, Y_{[n]}\right)
\end{aligned}
$$

where the last inequality follows from the independence between $W_{a}, S_{1}$, and $W_{b}$, and the fact that removing the conditioning does not decrease the entropy. Now, we have

$$
\begin{aligned}
\Delta_{t}^{(a)} & =\mathrm{I}\left(W_{a} ; Y_{t}, S_{t+1} \mid W_{b}, Y_{[t-1]}, S_{[t]}\right) \\
& =\mathrm{I}\left(W_{a} ; Y_{t} \mid W_{b}, Y_{[t-1]}, S_{[t]}\right) \\
& =\mathrm{H}\left(Y_{t} \mid W_{b}, Y_{[t-1]}, S_{[t]}\right)-\mathrm{H}\left(Y_{t} \mid W, Y_{[t-1]}, S_{[t]}\right) \\
& \leq \mathrm{H}\left(Y_{t} \mid W_{b}, S_{[t]}\right)-\mathrm{H}\left(Y_{t} \mid W, S_{[t]}\right) \\
& =\mathrm{I}\left(W_{a} ; Y_{t} \mid W_{b}, S_{[t]}\right),
\end{aligned}
$$

where the inequality above follows from the fact that $\mathrm{H}\left(Y_{t} \mid W_{b}, Y_{[t-1]}, S_{[t]}\right) \leq \mathrm{H}\left(Y_{t} \mid W_{b}, S_{[t]}\right)$ since removing the conditioning does not decrease the entropy, and that $\mathrm{H}\left(Y_{t} \mid W, Y_{[t-1]}, S_{[t]}\right)=\mathrm{H}\left(Y_{t} \mid W, S_{[t]}\right)$ due to absence of output feedback. Observe that, since, conditioned on $W_{b}$ and $S_{[t]}\left(\right.$ hence, on $\left.X_{t}^{(b)}\right), W_{a}-X_{t}^{(a)}-Y_{t}$ forms a Markov chain, the data processing inequality implies that

$$
\mathrm{I}\left(W_{a} ; Y_{t} \mid W_{b}, S_{[t]}\right) \leq \mathrm{I}\left(X_{t}^{(a)} ; Y_{t} \mid X_{t}^{(b)}, S_{[t]}\right) .
$$

By combining (21), (22), (23), and (24), one gets

$$
\begin{aligned}
R_{a} & \leq \frac{1}{n} \log \left|\mathcal{W}_{a}\right| \\
& \leq \frac{1}{n(1-\varepsilon)} \sum_{1 \leq t \leq n} \mathrm{I}\left(X_{t}^{(a)} ; Y_{t} \mid X_{t}^{(b)}, S_{[t]}\right)+\frac{\mathrm{H}(\varepsilon)}{n(1-\varepsilon)} \\
& \leq \frac{1}{n} \sum_{1 \leq t \leq n} \mathrm{I}\left(X_{t}^{(a)} ; Y_{t} \mid X_{t}^{(b)}, S_{[t]}\right)+\eta(\varepsilon) \\
& =\sum_{\boldsymbol{\sigma} \in \mathcal{S}^{(n)}} \alpha_{\boldsymbol{\sigma}} \mathrm{I}\left(X_{t}^{(a)} ; Y_{t} \mid X_{t}^{(b)}, S_{t}, S_{[t-1]}=\boldsymbol{\sigma}\right)+\eta(\varepsilon),
\end{aligned}
$$

which proves (14). 
In the same way, by reversing the roles of encoder $a$ and $b$, one obtains (15).

For $t \geq 1$, let us fix some string $\boldsymbol{\sigma} \in \mathcal{S}^{t-1}$, and focus our attention on the conditional mutual information terms $\mathrm{I}\left(X_{t} ; Y_{t} \mid S_{t}, S_{[t-1]}=\boldsymbol{\sigma}\right), \mathrm{I}\left(X_{t}^{(a)} ; Y_{t} \mid X_{t}^{(b)}, S_{t}, S_{[t-1]}=\sigma\right)$, and $\mathrm{I}\left(X_{t}^{(b)} ; Y_{t} \mid X_{t}^{(a)}, S_{t}, S_{[t-1]}=\boldsymbol{\sigma}\right)$, appearing in the rightmost sides of (13), (14), and (15), respectively. Clearly, the three of these quantities depend only on the joint conditional distribution of current channel state $S_{t}$, input $X_{t}$, and output $Y_{t}$, given the past state realization $S_{[t-1]}=\sigma$. Hence, the key step now consists in showing that

$$
\nu_{\boldsymbol{\sigma}}(s, x, y):=\mathbb{P}\left(S_{t}=s, X_{t}=x, Y_{t}=y \mid S_{[t-1]}=\boldsymbol{\sigma}\right)
$$

factorizes as in (4). This is proved in Lemma 6 below.

For $x_{i} \in \mathcal{X}_{i}, v_{i} \in \mathcal{V}_{i}$, and $\sigma \in \mathcal{S}^{t-1}$, let us consider the set $\Upsilon_{\boldsymbol{\sigma}}^{(i)}\left(x_{i}, v_{i}\right) \subseteq \mathcal{W}_{i}$,

$$
\Upsilon_{\boldsymbol{\sigma}}^{(i)}\left(x_{i}, v_{i}\right):=\left\{w_{i}: \phi_{t}^{(i)}\left(w_{i}, q_{i}\left(\sigma_{1}\right), \ldots, q\left(\sigma_{t-1}\right), v_{i}\right)=x_{i}\right\}
$$

and the probability distribution $\pi_{\boldsymbol{\sigma}}^{(i)}\left(\cdot \mid v_{i}\right) \in \mathcal{P}\left(\mathcal{X}_{i}\right)$,

$$
\pi_{\boldsymbol{\sigma}}^{(i)}\left(x_{i} \mid v_{i}\right):=\sum_{w_{i} \in \Upsilon_{\boldsymbol{\sigma}}^{(i)}\left(x_{i}, v_{i}\right)}\left|\mathcal{W}_{i}\right|^{-1} .
$$

Lemma 6: For every $1 \leq t \leq n, \boldsymbol{\sigma} \in \mathcal{S}^{t-1}, s \in \mathcal{S}, x_{a} \in \mathcal{X}_{a}$, and $x_{b} \in \mathcal{X}_{b}$,

$$
\begin{aligned}
& \nu_{\boldsymbol{\sigma}}(s, x, y) \\
& \quad=P(s) \pi_{\boldsymbol{\sigma}}^{(a)}\left(x_{a} \mid q_{a}(s)\right) \pi_{\boldsymbol{\sigma}}^{(b)}\left(x_{b} \mid q_{b}(s)\right) P(y \mid s, x) .
\end{aligned}
$$

Proof: First, observe that

$$
\begin{aligned}
\boldsymbol{\nu}_{\boldsymbol{\sigma}}(s, x, y) & =\mathbb{P}\left(S_{t}=s \mid S_{[t-1]}=\boldsymbol{\sigma}\right) \nu_{\boldsymbol{\sigma}}(x \mid s) P(y \mid s, x) \\
& =P(s) \nu_{\boldsymbol{\sigma}}(x \mid s) P(y \mid s, x)
\end{aligned}
$$

where $\nu_{\boldsymbol{\sigma}}(x \mid s):=\mathbb{P}\left(X_{t}=x \mid S_{[t]}=(\boldsymbol{\sigma}, s)\right)$. The former of the equalities in (27) follows from (1), while the latter is implied by the assumption that the channel state sequence is i.i.d.

Now, recall that, for $i \in\{a, b\}$, the current input satisfies $X_{t}^{(i)}=\phi_{t}^{(i)}\left(W_{i}, V_{[t]}^{(i)}\right)$. For $w \in \mathcal{W}$, let $\xi_{w}:=\mathbb{P}\left(X_{t}=x \mid\right.$ $\left.S_{[t]}=(\boldsymbol{\sigma}, s), W=w\right)$. Then,

$$
\begin{aligned}
\nu_{\boldsymbol{\sigma}}(x \mid s) & =\sum_{w} \xi_{w} \mathbb{P}\left(W=w \mid S_{[t]}=(\boldsymbol{\sigma}, s)\right) \\
& =\sum_{w}\left|\mathcal{W}_{a}\right|^{-1}\left|\mathcal{W}_{b}\right|^{-1} \xi_{w} \\
& =\sum_{w_{a} \in \Upsilon_{\boldsymbol{\sigma}}^{(a)}\left(x_{a}, q_{a}(s)\right)}\left|\mathcal{W}_{a}\right|^{-1} \sum_{w_{b} \in \Upsilon_{\boldsymbol{\sigma}}^{(b)}\left(x_{b}, q_{b}(s)\right)}\left|\mathcal{W}_{b}\right|^{-1} \\
& =\pi_{\boldsymbol{\sigma}}^{(a)}\left(x_{a} \mid q_{a}(s)\right) \pi_{\boldsymbol{\sigma}}^{(b)}\left(x_{b} \mid q_{b}(s)\right),
\end{aligned}
$$

the second inequality above following from the mutual independence of $S_{[t]}, W_{a}$, and $W_{b}$. The claim now follows from (27) and (28).

Let us now fix an achievable rate pair $R=\left(R_{a}, R_{b}\right)$. By choosing $(R, n, \varepsilon)$-codes for arbitrarily small $\varepsilon>0$, the in- equalities (13), (14), and (15), together with (10) and (12), imply that $\left(R_{a}, R_{b}\right)$ can be approximated by convex combinations of rate pairs (indexed by $\boldsymbol{\sigma} \in \mathcal{S}^{(n)}$ ) satisfying (3) for joint stateinput-output distributions as in (25). Hence, any achievable rate pair $R$ belongs to $\overline{\mathrm{CO}}\left(\cup_{\pi} \mathcal{R}(\pi)\right)$.

Remark 1: For the validity of the arguments above, two critical steps were (27), where the hypothesis of i.i.d. channel state sequence has been used, and (28), which only relies on the mutual independence of $W$ and $S_{[t]}$, this being a consequence of the assumption of absence of intersymbol interference. In particular, the key point in (27) is the fact that the past state realization $\boldsymbol{\sigma}$ appears in $\nu_{\boldsymbol{\sigma}}$ only and not in $P\left(S_{t}\right)$.

Remark 2: For the validity of the arguments above, it is critical that the receiver observes the channel state. More in general, it would suffice that the state information available at the decoder contains the one available at the two transmitters. In this way, the decoder does not need to estimate the coding policies used in a decentralized time-sharing.

\section{Extensions to Channels With Memory and CONCLUDING REMARKS}

The present paper has dealt with the problem of reliable transmission over finite-state multiple-access channels with asymmetric, partial channel state information at the encoders. A single-letter characterization of the capacity region has been provided in the special case when the channel state is a sequence of independent and identically distributed random variables.

It is worth commenting to which extent the results above can be generalized to channels with memory. Let us consider the case when the channel state sequence $\left\{S_{t}: t=1,2, \ldots\right\}$ forms a Markov chain with transition probabilities $\mathbb{P}\left(S_{t+1}=s_{+} \mid\right.$ $\left.S_{t}=s\right)=P\left(s_{+} \mid s\right)$ which are stationary and satisfy the strongly mixing condition $P\left(s_{+} \mid s\right)>0$ for all $s, s_{+} \in \mathcal{S}$. Further, assume that there is no intersymbol interference, i.e., $\left\{S_{t}: t=1,2, \ldots\right\}$ is independent from the message $W$, and that the state process is observed through quantized observations $V_{t}^{(i)}=q_{i}\left(S_{t}\right)$, as discussed earlier.

For the generation of optimal policies in a multiperson optimization problem, whenever a dynamic programming recursion via the construction of a Markov Chain with a fixed state space is possible (see [19] for a review of information structures in decentralized control), the optimization problem is computationally feasible and the problem is said to be tractable. In a large class of decentralized control problems, however, one faces intractable optimization problems. Let us elaborate on this further.

In team decision problems, one may assume that there is an a priori agreement among the decentralized decision makers on who will do what, when the random variables take place. This approach is based on Witsenhausen's equivalent model for discrete stochastic control [18], and makes the point that, indeed, all dynamic team problems are essentially static, with a much larger state space.

In the case of finite-state multiple-access channels with independent and identically distributed state sequences, by first showing that the past information is irrelevant, we observed that we could limit the memory space on which the dynamic opti- 
mization is performed. This is because, as observed in Remark 1 , in the rightmost side of (27) the past state realization $\sigma$ affects only the control $\nu_{\boldsymbol{\sigma}}(x \mid s)$, but not the current state distribution $P\left(S_{t}\right)$. In contrast, when the state sequence is a Markov chain, the past state realization $\boldsymbol{\sigma}$ does affect both the control $\nu_{\boldsymbol{\sigma}}(x \mid s)$ as well as the current state distribution $P\left(S_{t} \mid S_{[t-1]}=\boldsymbol{\sigma}\right)$. It is exactly such a joint dependence which prevents the proof presented here to be generalized to the Markov case.

Let us have a brief discussion for the case where there is only one transmitter. In this case, the conditional probability distribution of the state given the observation history, $\Pi_{t}(\cdot):=$ $\mathbb{P}\left(S_{t}=\cdot \mid V_{[t]}\right) \in \mathcal{P}(\mathcal{S})$, can be shown to be a sufficient statistic, i.e., the optimal coding policy can be shown to depend on it only. As a consequence, the optimization problem is tractable. Such a setting was studied in [20], where the following single-letter characterization was obtained for the capacity of finite-state single-user channels with quantized state observation at the transmitter and full state observation at the receiver:

$$
C:=\int_{\mathcal{P}(\mathcal{S})} \mathrm{d} \tilde{P}(\pi) \sup _{P(X \mid \pi)}\left\{\sum_{s} I(X ; Y \mid s, \pi) \tilde{P}(s \mid \pi)\right\}
$$

where the supremization runs over conditional input distributions $P(X \mid \pi) \in \mathcal{P}(\mathcal{X})$, and where $\tilde{P}(s, \pi):=\tilde{P}(s \mid \pi) \tilde{P}(\pi)$ denotes the asymptotic joint distribution of the state $S_{t}$ and its estimate $\Pi_{t}$, existence and uniqueness of which are ensured by the strong mixing condition.

For finite-state multiple-access channels with memory, a similar approach can successfully be undertaken only if the state observation is symmetric, namely if $q_{a}=q_{b}$. Indeed, in this case, the conditional state estimation $\Pi_{t}(\cdot)=\mathbb{P}\left(S_{t}=\cdot \mid V_{[t]}^{(a)}\right)=$ $\mathbb{P}\left(S_{t}=\cdot \mid V_{[t]}^{(b)}\right)$ can be shown to be a sufficient statistic, and a single-letter characterization of the capacity region can be proved.

However, for the general case when the channel state sequence has memory and the state observation is asymmetric (i.e., $q_{a} \neq q_{b}$ ), the construction of a Markov chain (which would not incur a loss in performance) is not straightforward. The conditional measure on the channel state is no longer a sufficient statistic: In particular, if one adopts a team decision based approach, where there is a fictitious centralized decision maker, this decision maker should make decisions for all the possible memory realizations, that is the policy is to map the variables $\left(W, V_{[t]}^{(a)}, V_{[t]}^{(b)}\right)$ to $\left(X_{t}^{(a)}, X_{t}^{(b)}\right)$ decentrally, and the memory cannot be truncated, as every additional bit is essential in the construction of an equivalent Markov chain to which the Markov Decision Dynamic Program can be applied; both for the prediction on the channel state as well as the belief of the coders on each other's memory. Let us also elaborate a discussion in view of common knowledge of Aumann [1]: Information between two decision makers is common knowledge if it is measurable with respect to the sigma-fields generated by both of the local information variables at the decision makers. It is not usual in practical applications that all the local information is common knowledge. In such scenarios, one approach is to have the decision makers compute the conditional probability measures for the exogenous random variables and the actions of other decision makers for generating their optimal actions. For example, in the context of our problem in the paper, if we look for such person-by-person optimal policies, a policy of one of the encoders (say Encoder $a$ ) which uses the past will force the other encoder (Encoder $b$ ) to also use the past to second-guess the action of Encoder $a$, which requires the use of a policy with memory. Thus, adopting a person-by-person policy does not lead to useful structural results, in our context.

We instead adopted Witsenhausen's equivalent model to generate team policies, as also elaborated in [19], by having the encoders agree on which policies to adopt before random variables are realized. The approach in our paper showed that we can obtain a direct result when the channel state sequence is memoryless. However, when the channel state has memory, the past information provides useful information which is important for estimating the future channel states. As such, we cannot avoid the use of the information on the past channel state realizations. If one is to construct an equivalent state based on which coding policies are generated, the equivalent state needs to keep growing with time: The discussion in [6] provides such a block-level characterization and it seems we cannot go beyond this due to the nontractability of the optimization problem. We note that if the encoders can exchange their past observations with a fixed delay, if they can exchange their observations periodically, or if they can exchange their beliefs at every time stage, then the optimization problem will be tractable.

One question of important practical interest is the following: If the channel transitions form a Markov chain, which is mixing fast, is it sufficient to use a finite memory construction for practical purposes? This is currently being investigated.

\section{REFERENCES}

[1] R. J. Aumann, "Agreeing to disagree," Ann. Stat., vol. 4, pp. 1236-1239, 1976.

[2] G. Caire and S. Shamai, "On the capacity of some channels with channel state information," IEEE Trans. Inf. Theory, vol. 45, pp. 2007-2019, 1999.

[3] Y. Cemal and Y. Steinberg, "Multiple access channel with partial state information at the encoders," IEEE Trans. Inf. Theory, vol. 51, pp. 3992-4003, 2005.

[4] J. Chen and T. Berger, "The capacity of finite-state markov channels with feedback," IEEE Trans. Inf. Theory, vol. 51, pp. 780-789, 2005.

[5] I. Csiszar and J. Körner, Information Theory: Coding Theorems For Discrete Memoryless Systems. Budapest, Hungary: Akademiai Kiado, 1981

[6] A. Das and P. Narayan, "Capacities of time-varying multiple-access channels with side information," IEEE Trans. Inf. Theory, vol. 48, no. 1, pp. 4-25, Jan. 2002.

[7] S. I. Gelfand and M. S. Pinsker, "Coding for channel with random parameters," Prob. Control Inf., vol. 9, pp. 19-31, 1980.

[8] A. Goldsmith and P. Varaiya, "Capacity of fading channels with channel side information," IEEE Trans. Inf. Theory, vol. 43, no. 6, pp. 1986-1992, Nov. 1997.

[9] S. A. Jafar, "Capacity with causal and non-causal side informationa unified view," IEEE Trans. Inf. Theory, vol. 52, pp. 5468-5475, 2006.

[10] M. Medard and R. Srikant, "Capacity of nearly-decomposable markov chains under asymmetric receiver-sender side information," IEEE Trans. Inf. Theory, vol. 52, pp. 3052-3062, 2006.

[11] H. Permuter, T. Weissman, and J. Chen, "New bounds for the capacity region of the finite-state multiple access channel," in Proc. IEEE Int. Symp. Inf. Theory, Toronto, ON, Canada, Jun. 2008, pp. 394-398.

[12] M. Salehi, "Capacity and coding for memories with real-time noisy defect information at encoder and decoder," Proc. Inst. Elec. Eng. Pt., vol. 139, pp. 113-117, Apr. 1992. 
[13] C. Shannon, "Channels with side information at the transmitter," IBM J. Res. Develop., vol. 2, pp. 289-293, 1958.

[14] S. Tatikonda and S. K. Mitter, "The capacity of channels with feedback," IEEE Trans. Inf. Theory, vol. 55, no. 1, pp. 323-349, Jan. 2009.

[15] S. Tatikonda, S. Yang, and A. Kavcic, "Feedback capacity of finitestate machine channels," IEEE Trans. Inf. Theory, vol. 51, pp. 799-810, 2005.

[16] S. Verdú and T. S. Han, "A general formula for channel capacity," IEEE Trans. Inf. Theory, vol. 40, no. 4, pp. 1147-1157, Jul. 1994.

[17] H. Viswanathan, "Capacity of markov channels with receiver CSI and delayed feedback," IEEE Trans. Inf. Theory, vol. 45, no. 2, pp. 761-771, Mar. 1999.

[18] H. S. Witsenhausen, "Equivalent stochastic control problems," Math. Control Signals Syst., vol. 1, pp. 3-11, 1988.

[19] S. Yüksel, "Stochastic nestedness and the belief sharing information pattern," IEEE Trans. Autom. Control, vol. 55, pp. 2773-2786, 2009.

[20] S. Yüksel and S. Tatikonda, "Capacity of markov channels with partial state feedback," in Proc. IEEE Int. Symp. Inf. Theory (ISIT), Nice, France, Jun. 2007, pp. 1861-1865.
Giacomo Como received the B.Sc., M.S., and Ph.D. degrees in applied mathematics from Politecnico di Torino, Italy, in 2002, 2004, and 2008, respectively.

$\mathrm{He}$ is currently a Postdoctoral Associate at the Laboratory for Information and Decision Systems, Massachusetts Institute of Technology, Cambridge. In 2006-2007 he was Visiting Assistant in Research at the Department of Electrical Engineering, Yale University. His current research interests include the mathematics of information and control theory, network science, and coding theory.

Serdar Yüksel received the B.Sc. degree in electrical and electronics engineering from Bilkent University, Ankara, Turkey, in 2001 and the M.S. and $\mathrm{Ph} . \mathrm{D}$. degrees in electrical and computer engineering from the University of Illinois at Urbana-Champaign in 2003 and 2006, respectively.

He was a Postdoctoral Researcher at Yale University for a year before joining Queen's University, Kingston, ON, Canada, as an Assistant Professor of Mathematics and Engineering in the Department of Mathematics and Statistics. His research interests are in stochastic and decentralized control, information theory, and applied probability.

Dr. Yüksel serves on the IFAC (International Federation of Automatic Control) Committee on Stochastic Systems. 